https://doi.org/10.21670/ref.1806006

Artículos

\title{
Cooperación local binacional: La cuenca internacional del Río Tijuana
}

\section{Local binational cooperation: The Tijuana River international watershed}

\author{
José Luis Castro Ruiz * (iD) https://orcid.org/0000-0002-1601-2175 \\ Stephen P. Mumme ${ }^{b}$ iD https://orcid.org/0000-0002-4373-8430 \\ Kimberly Collins ${ }^{c}$ (i) https://orcid.org/0000-0003-0770-0416
}

Recibido el 12 de mayo de 2017. Aceptado el 8 de diciembre de 2017. Publicado el 12 de marzo de 2018.

${ }^{*}$ Autor para correspondencia: José Luis Castro Ruiz, correo electrónico: jlcastro@colef.mx

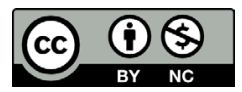

Esta obra está protegida bajo una Licencia Creative Commons Atribución-NoComercial 4.0 Internacional.

\begin{abstract}
${ }^{\text {a }}$ El Colegio de la Frontera Norte, Departamento de Estudios Urbanos y del Medio Ambiente, Monterrey, Nuevo León, México, correo electrónico: jlcastro@colef.mx

${ }^{\mathrm{b}}$ Colorado State University, Department of Political Science, Estados Unidos de América, correo electrónico: smumme@colostate.edu

${ }^{\mathrm{c}}$ California State University, San Bernardino, Estados Unidos de América, correo electrónico: kimberly@csusb.edu
\end{abstract}

\section{Resumen}

Se examina la experiencia de iniciativas de colaboración ambiental a nivel local binacional en la Cuenca del Río Tijuana, y la participación gubernamental de México y Estados Unidos en esos ámbitos. El análisis comprende cuatro puntos principales: las características y problemáticas ambientales de la cuenca; las iniciativas locales binacionales en las últimas décadas; la participación gubernamental en dicha experiencia, en el contexto de las diferencias estructurales entre ambos países, así como algunas reflexiones en el marco del debate global de la importancia de los niveles locales en cuencas transfronterizas. Se ilustra el potencial que iniciativas locales como las estudiadas ofrecen para complementar la búsqueda de soluciones cooperativas a los problemas hídricos comunes entre ambos países. Se concluye que el Acta 320 de la cila-IBWC constituye una oportunidad única para las instancias gubernamentales mexicanas para instrumentar mecanismos operativos que faciliten la interacción con sus contrapartes sociales y gubernamentales a los niveles sub-nacionales.

Palabras clave: Cuenca del Río Tijuana, región Tijuana-San Diego, Tratado de Aguas de 1944, cooperación binacional, política hídrica nacional.

\section{Abstract}

The experience behind local environmental bi-national collaborative initiatives in the Tijuana river watershed is examined, and the governmental participation from Mexico and the U.S. in those spheres. The analysis encompasses four main topics:

CÓMO CITAR: Castro, J. L., Mumme, S. P. y Collins, K. (2018). Cooperación local binacional: La cuenca internacional del Río Tijuana [Local binational cooperation: The Tijuana river international watershed]. Estudios Fronterizos, 19, e006. https://doi. org/10.21670/ref.1806006 
the characteristics and environmental problems of the watershed; the local bi-national initiatives undertaken on the last decades; the governmental involvement in that experience, in the context of the structural differences between the two countries, and some reflections from the perspective of the global debate on the importance of local levels in trans-boundary watersheds. The results illustrate the potential that local initiatives as those studied, presents to complement the search for cooperative solutions to the common bi-national water problems. It is concluded that Minute 320 from CILA-IBWC represents a unique opportunity for the Mexican governmental instances to implement operative mechanisms to facilitate the interaction with their social and governmental counterparts at the sub-national levels.

Keywords: Tijuana River Watershed, San Diego-Tijuana region, 1944 Water Treaty, bi-national cooperation, national water policy.

\section{Introducción}

El Río Tijuana, ubicado en la región fronteriza Tijuana-San Diego, a diferencia de los otros sistemas superficiales referidos en el Tratado sobre distribución de aguas internacionales celebrado entre los Estados Unidos Mexicanos y los Estados Unidos de América de 1944, tiene la distinción de ser la única corriente cuyas aguas permanecen sin distribuirse binacionalmente, a pesar de la existencia del artículo, que establece expresamente los lineamientos de autoridad en torno al manejo bilateral de sus aguas, (Tratado sobre distribución de aguas internacionales, 1944, art. 16), ${ }^{1}$ por lo que es más conocido por sus problemas crónicos de contaminación por aguas residuales sin tratar que por sus recursos naturales. En contraste con esta condición, en las últimas décadas su cuenca se ha caracterizado por ser objeto de diferentes iniciativas a nivel local binacional, únicas en el contexto fronterizo, las que han surgido bajo el objetivo de encontrar soluciones a su creciente problemática urbana y ambiental, y que adquirieron una importancia clave después del año 2000 (Brown, Castro, Lowery y Wright, 2003). La culminación de esta creciente preocupación pública y trabajo a favor de la cuenca se reflejó en la firma del Acta 320 entre ambos países vía la Comisión Internacional de Límites y Aguas (ciLA) y su contraparte norteamericana, la International Boundary and Water Commission (IBWC), un acuerdo marco que asegura una mayor atención para un amplio rango de problemas asociados con el grado de urbanización de la cuenca, promoviendo un cambio en el patrón histórico de abandono binacional de la misma (cILA, 2015).

Una característica sobresaliente de dichas actividades ha sido la participación gubernamental en losámbitos subnacionales, la que sin embargo ha sido marcadamente estadounidense. En el caso de los recursos hídricos, el papel de las instancias mexicanas se ha limitado al trabajo operativo de la cILA, debido a que la Ley de Aguas Nacionales

\footnotetext{
${ }^{1}$ El artículo 16 del Tratado sobre distribución de aguas internacionales celebrado entre los Estados Unidos Mexicanos y los Estados Unidos de América, conocido también como Tratado de Aguas, fue escrito como un acuerdo marco cubriendo un rango de asuntos que afectan a la Cuenca del Río Tijuana, donde se estipulan las responsabilidades de la CILA y su contraparte estadounidense IBWC ante ambos gobiernos en relación con: 1) la distribución equitativa de las aguas del Río Tijuana; 2) almacenamiento y control de avenidas y desarrollo de diferentes usos de agua; 3) planes para la construcción de obras propuestas y la división de sus costos entre ambos países; y 4) recomendaciones sobre el papel de la Comisión y sus secciones nacionales en la operación y mantenimiento de las obras construidas.
} 
no contempla las cuencas transfronterizas internacionales. El objetivo del presente estudio es examinar esta experiencia y sus beneficios potenciales como un importante medio de coordinación transfronteriza que complemente las vías tradicionales en que ambos países abordan y buscan soluciones a sus problemas hídricos comunes. El trabajo se encuentra dividido del siguiente modo: primero se describe sucintamente la situación del Río Tijuana en el marco del Tratado de Aguas de 1944; enseguida, las características de su cuenca y las problemáticas que enfrenta en la actualidad. Después, se abordan algunas de las iniciativas locales binacionales más importantes que han tenido lugar en el seno de la cuenca desde la década de 1990, como una clara evidencia del creciente interés regional por avanzar soluciones integrales y cooperativas de manejo para este río, incluso proyectos y acciones en el marco de los acuerdos bilaterales que surgieron en forma posterior al Tratado de Aguas de 1944, los que han puesto un énfasis marcado en las capacidades locales. A continuación, se aborda el papel de la participación gubernamental en esta experiencia local en el contexto de las diferencias estructurales, tanto institucionales como de política hídrica entre ambos países, y cuyo balance es desfavorable para México. El último apartado presenta algunas reflexiones sobre la experiencia de la Cuenca del Río Tijuana (CRT) en el contexto del debate global en torno a la importancia que han adquirido los niveles locales en cuencas transfronterizas, así como los beneficios que implica la existencia de dichas capacidades locales en un marco de cooperación binacional entre ambos países. Entre los puntos más sobresalientes que cabe mencionar están las implicaciones para México de la firma del Acta 320 de la cILA, como una importante vía para avanzar en modelos de manejo cooperativo y sustentable de los recursos hídricos compartidos en la frontera norte, en forma complementaria a las instancias tradicionales.

\section{El Río Tijuana en el contexto del Tratado de Aguas de 1944}

El desafío que implica el manejo binacional del Río Tijuana debe situarse en el marco del Tratado de Aguas de 1944, así como de otros acuerdos bilaterales que lo han antecedido sobre la diplomacia y práctica en torno a las corrientes compartidas a lo largo de la frontera México-Estados Unidos. Desde el establecimiento de la frontera común por virtud de los tratados de Guadalupe-Hidalgo y Gadsden, ${ }^{2}$ ambos países se han dado a la tarea de elaborar una serie de tratados y acuerdos subsidiarios, los que constituyen hoy en día uno de los regímenes más institucionalizados de manejo de aguas transfronterizas en el mundo. Un marco que ha sido descrito frecuentemente como la estructura soberana vital que sustenta las relaciones de amistad en este campo entre ambos países (Friedkin, 1965; McBride, 1981).

Esta estructura institucional, particularmente desde la firma del Tratado de Aguas de 1944, ha encontrado su adaptación a un amplio rango de problemas bilaterales en torno al agua a lo largo de la frontera internacional, extendiendo su régimen al manejo de los cursos de agua no referidos en el tratado, así como a los problemas de calidad del agua en ríos y corrientes transfronterizas. En la actualidad, sin embargo, muchos de estos problemas permanecen ausentes de un abordaje operativo, y la

${ }^{2}$ El tratado de Gadsden en 1853 culminó la venta del territorio de La Mesilla de México a Estados Unidos, definiendo la actual línea fronteriza entre ambos países. 
falta de autoridad expresa dentro del tratado se suma a la dificultad de avanzar en la búsqueda de soluciones, trátese de la problemática de las aguas subterráneas o de las corrientes no referidas en el tratado, como son por ejemplo los ríos Santa Cruz y San Pedro en la frontera entre Sonora y Arizona. El Río Tijuana es una excepción a esta situación, ya que como se ha mencionado sus aguas permanecen sin distribuirse, a pesar de la existencia de acuerdos binacionales concretos plasmados en el artículo 16 en el Tratado de Aguas de 1944; acuerdos que indudablemente se sustentaban en un conocimiento de sus condiciones de oferta y demanda existentes, así como del crecimiento futuro esperado (Samaniego, 2006, p. 358). ${ }^{3}$

\section{Descripción de la cuenca}

El Río Tijuana está formado por diferentes corrientes originadas en México y Estados Unidos, principalmente el Río de Las Palmas en México y el Cottonwood Creek en la parte estadounidense de la cuenca. Ambos afluentes convergen dentro de la zona urbana de la ciudad de Tijuana, $16 \mathrm{~km}$ al este del océano Pacífico, y drenan en el mar cerca de una reserva ecológica al norte de la línea fronteriza. La cuenca comprende una superficie de $4430 \mathrm{~km}^{2}$, ubicándose dos terceras partes en territorio mexicano (Figura 1).

Debido a las condiciones climáticas semiáridas de esta zona, considerada de tipo mediterráneo, la mayoría de las corrientes de agua son intermitentes, con caudales máximos entre los meses de noviembre y abril. Los escurrimientos respectivos son captados por las presas de la cuenca en ambos países y por los acuíferos que suministran agua a comunidades locales; sin embargo, la mayor proporción del agua consumida en la cuenca (más de 80\%) proviene del Río Colorado a través del Acueducto Río Colorado-Tijuana (АRст) en el caso de Tijuana y Tecate, y por medio del Distrito Metropolitano de Aguas del Sur de California (Metropolitan Water District of Southern California [MWDSc]) desde el norte, en el caso de la parte estadounidense de la cuenca. Las fuentes subterráneas contribuyen en mayor proporción a las necesidades de la parte mexicana. En la ciudad de Tijuana la proporción de fuentes subterráneas es de $5 \%$, en tanto que en Tecate es de casi $30 \%$.

En 2010 más de millón y medio de personas habitaban dentro de los límites de la cuenca; de los cuales, aproximadamente $97 \%$ residen en la parte mexicana. Los centros urbanos de la cuenca concentran a más de $90 \%$ de la población, dándole una connotación marcadamente urbana a su problemática ambiental. Las proyecciones para la cuenca prevén un crecimiento de $100 \%$ en su población para el año 2030 (Institute for Regional Studies of the Californias [IRSC], 2005, pp. 17-18). En la porción mexicana de la misma, y de acuerdo con proyecciones del Consejo Nacional de Población (Conapo), los municipios de Tijuana y Tecate formarán parte de un área metropolitana con una población de alrededor de 2.33 millones en el mismo año, lo que representa un incremento de $30.5 \%$ sobre la estadística de 2010 (Conapo, 2013). La responsabilidad correspondiente a las demandas de infraestructura

\footnotetext{
${ }^{3} \mathrm{Al}$ ser considerada de menor importancia la necesidad de llegar a arreglos sobre la distribución de sus aguas, la atención al Río Tijuana se ha enfocado en buena medida al control de sus avenidas, mismas que, a pesar de los bajos escurrimientos anuales, han representado un problema recurrente en la región.
} 
y servicios será evidente, así como los impactos sobre la calidad de vida, fragmentación del hábitat, y la vida de las plantas y animales relacionados con el corredor natural que va desde las Montañas de Otay hasta el sureste de Tijuana (IRsc, 2005, pp. 17-18).

Figura 1: Cuenca del Río Tijuana

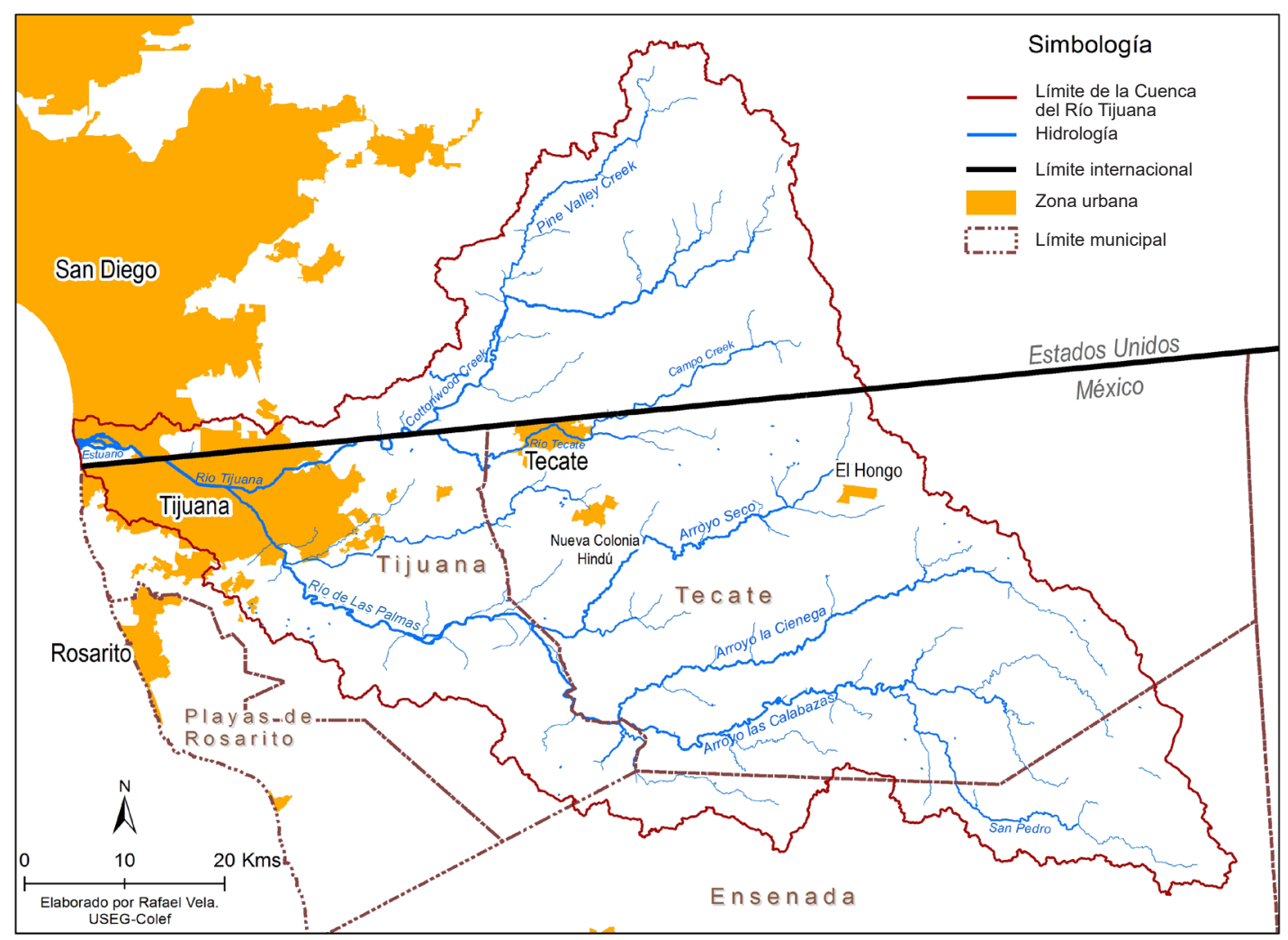

Fuente: Elaborado por Rafael Vela.

\section{Problemática hídrica de la cuenca}

La Cuenca del Río Tijuana enfrenta múltiples retos, producto básicamente del crecimiento urbano que ha tenido lugar dentro de sus límites. Entre los relacionados con los recursos hídricos se encuentra la oferta y distribución de estos, calidad del agua y saneamiento, necesidades de protección ecológica, inundaciones y control de avenidas y manejo de sedimentos. La mayoría de estos problemas requiere de un tratamiento binacional para su óptimo abordaje y manejo, con los consecuentes problemas logístico-diplomáticos (Mumme, Collins y Castro, 2014). 


\section{Oferta y calidad del agua y saneamiento}

La demanda histórica del agua en la cuenca ha ejercido una presión continua en la oferta disponible, llevando a las autoridades responsables a cada lado de la frontera a buscar formas alternas de asegurar el abasto futuro, entre las que la participación del Río Tijuana se ha desdibujado en su dimensión como un recurso binacional. En la porción estadounidense de la cuenca, las presas Barret y Morena han formado parte del sistema local de suministro de agua desde su construcción, a principios del siglo xx. En la década de 1940, la creciente demanda urbana llevó a la Autoridad de Aguas del Condado de San Diego (San Diego County Water Authority [sDCwA]) a importar agua del Río Colorado, a través del Distrito Metropolitano de Aguas (Metropolitan Water District [MwD]), lo que satisfizo entre $85 \%$ y $90 \%$ de las necesidades de dicha fuente. El resto de la política hídrica actual se centra en el potencial de almacenaje de agua subterránea del estuario del Río Tijuana, al norte de la línea internacional (City of San Diego, 2010). La ciudad de Tijuana, por otro lado, ha tenido que enfrentar históricamente la demanda producto de su explosivo crecimiento poblacional, lo que llevó a las autoridades del estado de Baja California a depender básicamente de la importación de agua del Río Colorado desde la década de 1980. No obstante, las referencias al Río Tijuana en el Programa Estatal de Agua para Baja California, 2008-2013 se limitan más bien a los problemas de contaminación provocados por las aguas residuales de Tijuana y los proyectos para tratarlos (Comisión Estatal del Agua de Baja California [CEA], 2008).

La rápida urbanización y las prácticas agrícolas son las principales fuentes contaminantes que amenazan a la CRT; a esto se agregan el crecimiento de los asentamientos humanos en la región de Tijuana y la industrialización del corredor fronterizo. El desarrollo de la industria maquiladora desde 1965 ha impactado la calidad del agua, en tanto que los asentamientos humanos sin conexión a la red urbana de drenaje a lo largo de los empinados cañones han contribuido a la erosión y acumulación tanto de basura como de aguas residuales sin tratar en los arroyos inferiores. De hecho, los escurrimientos de aguas de drenaje sin control a través de la frontera han sido un factor que ha afectado las relaciones binacionales por décadas (CILA, 1965).

En la parte norteamericana de la cuenca, la Junta Estatal de Control de Recursos Hídricos de California (California State Water Resources Control Board [CSWRCB]) tiene clasificado al Río Tijuana como una cuenca categoría uno, deteriorada, a causa de sus muchos problemas de contaminación (IRSC, 2005, p. 59). En la década de 1980 ambos países colaboraron en el proyecto de una Planta Internacional de Tratamiento de Aguas al norte de la línea fronteriza (CILA, 1985; 1990), cuya capacidad ha sido ampliada periódicamente, pero que no satisface las necesidades actuales (Saldaña, 2003).

\section{Biodiversidad y ecosistemas}

La CRT alberga una notable abundancia de vegetación y vida silvestre, y representa un espacio crítico para las rutas de aves en la costa del Pacífico (Michel, 2001). La zona de humedales protegidos que forman parte de la Reserva del Estuario del Río Tijuana (Tijuana River National Estuarine Research Reserve [TRNERR]) y su entorno se encuentran entre los pocos espacios de este tipo con baja alteración a lo largo 
de la costa californiana, como producto de un trabajo de conservación que se ha extendido por cerca de medio siglo (TRNERr, 2010). A pesar de estos esfuerzos y de una inversión considerable para apoyarlos, el estuario permanece vulnerable a procesos de erosión y sedimentación que son agravados por los asentamientos irregulares a lo largo de los cañones en la parte mexicana, y por la construcción de infraestructura de seguridad fronteriza en las inmediaciones del estuario de la parte estadounidense.

Si bien la situación ambiental del estuario es el problema más conocido del Río Tijuana, existen otros problemas que afectan la biodiversidad y que son resultado del desarrollo industrial y urbano en la cuenca, como es el caso de los afluentes que alimentan al río que proporcionan aún un hábitat de fauna y flora que no está presente en la zona urbana. Entre ellos, sobresale la subcuenca del Río Alamar, cercana al límite internacional, la que es reconocida como uno de los pocos sistemas riparios que sobreviven en la región de Tijuana, pero que es altamente vulnerable a los nuevos desarrollos en la misma. La Comisión Nacional del Agua (Conagua) ha puesto en marcha recientemente sus planes de canalización de la parte baja del río con el argumento de seguridad contra inundaciones y mejoramiento de la imagen urbana ("Comienza Última Etapa", 2015), sin considerar los impactos en los recursos biológicos y servicios ecosistémicos que aporta (Trejo y Castro, 2013).

\section{Lluvias, inundaciones y sedimentos}

La región Tijuana-San Diego se sitúa en la pendiente occidental de la costa californiana, donde los niveles de precipitación, cañones empinados y suelos arenosos se combinan para hacer a ambas ciudades vulnerables a inundaciones periódicas y a la acumulación de sedimentos en las partes bajas de los arroyos. Los primeros trabajos de infraestructura hídrica se centraron en controlar las corrientes de los ríos Cottonwood y Las Palmas; en la actualidad, el sistema de presas dentro de la cuenca capta $78 \%$ de los escurrimientos del Río Tijuana (Coastal, 2006). Los riesgos de inundaciones aguas abajo de las presas Barrett y Moreno en Cottonwood Creek, y del Carrizo y Rodríguez en el Río de Las Palmas son un problema serio, cuyos efectos reales se resienten en el estuario al final del Río Tijuana, como se mencionó anteriormente.

\section{Iniciativas de manejo binacional en la Cuenca del Río Tijuana}

Debido a los desafíos que enfrenta la CRT, esta ha sido escenario de importantes iniciativas locales y regionales de cooperación binacional, cuya historia se remonta a la década de 1990 bajo el liderazgo cooperativo del sector académico a ambos lados de la frontera (Brown et al., 2003). ${ }^{4}$ Un marco que fue fundamental para generar nexos

\footnotetext{
${ }^{4}$ Además del proyecto de la Cuenca del Río Tijuana, que buscaba abordar sus problemas ambientales, la Universidad Estatal de San Diego y El Colegio de la Frontera Norte se dieron a la tarea de desarrollar un sistema de información geográfica de la cuenca para su promoción entre las comunidades, el sector educativo y el de investigación científica.
} 
con grupos ciudadanos a nivel local, fue la creación de los Mecanismos de Enlace Fronterizo (BLM, por sus siglas en inglés) a lo largo de la frontera por parte de los niveles federales en ambos países en 1993 (Brown y Mumme, 2000). Un rasgo importante de estos mecanismos es la participación de los consulados generales de cada país como convocantes y facilitadores del diálogo binacional entre actores gubernamentales y la sociedad civil en torno a diversas problemáticas de la cuenca, incluyendo la calidad del agua y el drenaje sanitario y pluvial.

Dentro del marco del Mecanismo de Enlace Fronterizo existe un número de agencias en los tres niveles de gobierno de cada país, que conforman el Consejo Fronterizo del Agua (вwс, por sus siglas en inglés). Por parte del gobierno federal estadounidense están la sección correspondiente de la Comisión Internacional de Límites y Aguas y la Agencia de Protección Ambiental (Environmental Protection Agency [EPA]), además de otras instancias que participan esporádicamente. A nivel local están los gobiernos tribales, la Autoridad de Aguas de la Ciudad de San Diego, el condado y la ciudad de San Diego. En la parte mexicana las instancias federales participantes en el BwC incluyen a Conagua, cila y la Secretaría del Medio Ambiente y Recursos Naturales (Semarnat), incluyendo también a la Comisión Estatal de Servicios Públicos de Tijuana (CESPT), por parte del estado y al gobierno municipal de Tijuana. Finalmente, el trabajo del вwC se complementa con iniciativas en la parte estadounidense de la cuenca como el programa de manejo de sedimentos en el Cañón de Los Laureles (Heyn, Keane-Dengel, Lewis, Phillips y Virgilio, 2008). Aunado a ello, el Panel Regional de Control de Calidad del Agua de San Diego, la Comisión Costera y Conservación Costera son agencias que apoyan proyectos de calidad del agua y restauración ecológica en la parte baja y la zona del estuario de la CRT.

Posiblemente el esfuerzo de manejo de la cuenca más notable se dio a través de diferentes programas y acciones a nivel local, muchos de ellos en el ámbito no gubernamental. En el año 2003, y en el marco de una nueva autoridad del gobierno de California para desarrollar planes de manejo ripario a lo ancho de la cuenca, un grupo de investigadores de la Universidad Estatal de San Diego y de El Colegio de la Frontera Norte trabajaron conjuntamente en el proyecto de creación de un cuerpo consultivo binacional, el Consejo Consultivo Binacional de la CRT (BWAC), el cual integró a la academia, organizaciones no gubernamentales, representaciones de grupos indios, así como a otras entidades del sector privado, con el fin último de desarrollar un documento binacional de naturaleza integral que guiara los esfuerzos de evaluación y planeación en la cuenca. El reporte denominado Una Visión Binacional para la Cuenca del Río Tijuana (IRsc, 2005), identificó un amplio espectro de mejoras potenciales para la cuenca, e incluyó medidas, tales como la construcción de humedales, parques ecológicos y acciones de conservación. ${ }^{5}$ La agenda establecida por el Consejo sentó la base para la creación de un número creciente de organizaciones de la sociedad civil

\footnotetext{
${ }^{5}$ Entre las actividades de seguimiento que se desarrollaron a partir de la terminación del proyecto en marzo de 2005, estuvo la formación del Grupo de Trabajo de Agua como parte del Programa Frontera 2012, el subgrupo de trabajo de Ecología, así como el Comité Técnico del Agua creado bajo el Mecanismo de Enlace Fronterizo (BLM) dependiente del Comité de Oportunidades Regionales Binacionales (COBRO), un organismo perteneciente a la Asociación de Gobiernos de San Diego (San Diego Association of Goverments [SANDAG]). Los responsables y organizadores del proyecto han desplegado nuevas metas y acuerdos para continuar con el mismo, los que fueron plasmados en el Taller sobre Manejo de la Cuenca Binacional del Río Tijuana, realizado en Tijuana, B. C., los días 14-16 de mayo de 2013.
} 
que se han dado a la tarea de abordar temas específicos dentro de la cuenca (Mumme et al, 2014) ${ }^{6}$.

La trayectoria de proyectos referidos aquí, conforma una base importante de conocimientos y recursos para el abordaje de la problemática ambiental de la CRT. El trabajo e interés público que ha generado esta tendencia ha adquirido una dinámica paralela al desarrollo mismo de la región y su interdependencia binacional, en la búsqueda de lo que se mantiene como el principal desafío: trascender las múltiples iniciativas individuales y conexiones transfronterizas hacia esquemas más integrales de planeación que generen el suficiente apoyo gubernamental a ambos lados de la frontera. Es muy significativa e histórica en este sentido la reciente firma del Acta 320 de la CILA-IBWC, ya que establece un marco conceptual y de acciones que guía el trabajo de cooperación binacional en la problemática hídrica de la CRT (CILA, 2015), un evento que puede considerarse como la culminación del intenso trabajo de análisis y propuestas de opciones de política de los diferentes grupos de interés y actores no gubernamentales en ambas partes de la cuenca (Saxod, Castro, Silvan y Reyna, 2007). ${ }^{7}$

\section{Iniciativas en el marco de acuerdos bilaterales posteriores al Tratado de Aguas de 1944}

En las últimas décadas, el surgimiento de diferentes acuerdos y programas binacionales bajo el objetivo general de atender los problemas ambientales en la frontera común entre ambos países, significó una ampliación considerable de la cobertura y funciones de la CILA-IBWC, al abordar en forma más puntual la problemática hídrica, y buscar la integración de las comunidades fronterizas en los procesos de planeación y toma de decisiones. En ese contexto, la CRT ha sido objeto de programas que han operado con insumos locales a partir de la década de 1990 (Spalding, 1999).

Desde su adopción, en 1983, el Acuerdo de La Paz sobre cooperación ambiental binacional en la zona fronteriza — nombrado así por la ciudad porteña donde fue signado por los presidentes Miguel de la Madrid y Ronald Reagan— ha probado ser un marco fundamental de política para sustentar un número de iniciativas ambientales en torno al manejo de los ríos transfronterizos. Este acuerdo requiere de un proceso de consulta binacional en forma regular sobre cuestiones ambientales, y crea un mecanismo diplomático para establecer compromisos sustantivos y de procedimiento enfocados a problemas específicos de cada región en la zona fronteriza (Mumme y Collins, 2014). En 1985 uno de estos acuerdos accesorios, el Anexo I asociado al Acta 270 de la cILA, fortaleció la cooperación binacional en el problema de limpieza y saneamiento del Río Tijuana en la frontera internacional (cILA, 1985). El Acuerdo de La Paz también aportó las bases de política para los programas de cooperación

\footnotetext{
${ }^{6}$ Entre estas organizaciones resalta el trabajo de grupos binacionales como la Red de Educación Ambiental Binacional, el proyecto Bioregional de Educación Ambiental, Ja Jan, Los Niños, así como el Proyecto Fronterizo de Educación Ambiental, los que han concentrado su atención en los problemas de contaminación del río y su valor ecológico.

${ }^{7}$ Una de las características centrales del Acta 320 es la conformación de un Grupo Base Binacional (GBB), para coordinar la formulación de recomendaciones en torno a la CRT, en el que se plantea incorporar además de los diferentes niveles de gobierno, a representantes de organizaciones no gubernamentales de ambos países.
} 
ambiental binacional que desarrollaron ambos gobiernos a partir de 1991 hasta el actual programa Frontera 2020. En el caso de la CRT, este programa apoya el trabajo del Grupo Regional de Trabajo para Baja California-California (EPA, 2010) y el Grupo de Trabajo Binacional (Tijuana-San Diego) para la cuenca, ambos importantes instancias para abordar los problemas relacionados con la sustentabilidad ecológica y la salud pública.

Siguiendo el ejemplo de los programas emprendidos por agencias e instituciones como las referidas anteriormente, la sección estadounidense de la cILA-IBWC emprendió en 1999 una serie de foros ciudadanos a lo largo de la frontera binacional con la idea de integrar las opiniones y experiencia de personas y grupos interesados (stakeholders) en las comunidades fronterizas estadounidenses. En el caso de la región Tijuana-San Diego y del estuario del Río Tijuana, se abordó la problemática relacionada con el control de inundaciones y los flujos de aguas residuales de Tijuana y sus efectos en el área del estuario en la parte estadounidense (cıla Sección Norteamericana, s.f.). Un proyecto importante surgido de esta iniciativa fue el Tijuana River Valley Recovery Strategy con el objetivo de reducir las descargas del Río Tijuana hacia su parte final, y limpiarla de sedimientos y residuos (Tijuana River Valley Recovery Team, 2012). El modelo de estos foros ha comenzado recientemente a ser reproducido por la parte mexicana de la cila, a partir de la instalación del foro ciudadano en Tijuana, en agosto de 2014 (cila Sección Mexicana, 2014).

En 1993, en el marco del Tratado de Libre Comercio de América del Norte, surgieron la Comisión de Cooperación Ecológica Fronteriza (COCEF) y su asociado institucional, el Banco de Desarrollo de América del Norte (NADBank), con el propósito de facilitar el desarrollo y financiamiento de proyectos de infraestructura ambiental a lo largo de la frontera binacional. El trabajo de cocef a través de su asociación con gobiernos y comunidades locales ha mostrado su potencial para incrementar y reforzar las capacidades locales en los procesos de restauración ambiental y ecológica en la CRT, lo que la ha convertido en una importante vía para abordar el amplio espectro de problemas de la cuenca.

Entre los proyectos apoyados por COCEF-NADBANK se encuentra la construcción y mejora de infraestructura de saneamiento y tratamiento de aguas residuales en Tecate, Tijuana y el área del estuario del Río Tijuana, en la parte estadounidense de la cuenca. Uno de los proyectos sobresalientes fue la construcción de una planta en la zona del estuario en 2002, para tratar las aguas de desecho del Río Tijuana. En el caso de Tijuana y Tecate, el objetivo ha sido la ampliación de los sistemas existentes (NADBank, 2013, p. 6). Otro proyecto importante para el área de Tijuana fue el desarrollo de un Plan Maestro de Agua Potable y Saneamiento, que incluyó al municipio de Playas de Rosarito en 2003, y cuyo financiamiento provino de la EPA (CESPT, 2003).

\section{El papel gubernamental en los ámbitos subnacionales}

Un elemento que ha condicionado de manera relevante la participación gubernamental en la experiencia binacional descrita aquí, son las diferencias estructurales que existen entre ambos países, las que no solo determinan esquemas de gestión diferentes a cada lado de la frontera, sino la forma en que los niveles gubernamentales involucrados se relacionan con sus contrapartes transfronterizas. La estructura política de los Estados 
Unidos está fundamentada en un arreglo federal que se origina en la Constitución, por el que el gobierno central y los gobiernos estatales dividen el ejercicio del poder en diferentes temas. En la práctica y en particular sobre el manejo del agua, el modelo federalizado estadounidense despliega una fragmentación considerable que abarca a los niveles locales con competencia en el tema. En el caso de México, la Constitución otorga a la nación la propiedad del recurso, el cual es regulado y manejado por el estado. En términos operativos, y pese a las modificaciones constitucionales, como fue la reforma municipal en la década de 1980, la estructura político administrativa en el país es básicamente centralizada.

Las diferencias anteriores son ejemplificadas claramente en la CRT. En la parte estadounidense, la Agencia de Protección Ambiental (EPA, por sus siglas en inglés) es la principal agencia federal responsable de la gestión de la calidad del agua bajo los preceptos de la Clean Water Act. La oficina regional para esta región (Región 9) delega autoridad a nivel estatal en la Junta de Control de Recursos Hídricos (California State Water Resourses Control Board [CASwrCb]) la cual es gobernada por la Porter-Cologne Water Quality Act de naturaleza estatal. Las responsabilidades de este órgano son delegadas a su vez en nueve Juntas de Control Regional de Calidad del Agua (California Regional Water Quality Control Board [CARWQCB]). La Región 9 es la CARWQCB particular para el Condado de San Diego y la parte estadounidense de la cuenca. En forma similar muchas otras reglamentaciones federales, estatales y locales inciden para restringir efectos adversos en el medio ambiente en la parte estadounidense de la cuenca, incluyendo el aire, tierra, recursos culturales e impactos socioeconómicos (IRSC, 2005, p. 190).$^{8}$

Para el caso de México, las reformas a la Ley de Aguas Nacionales de 2004 confieren a la Conagua, autoridad principal en la materia, la gestión del agua en los niveles federal y regional a través de regiones hidrológico-administrativas, mediante los organismos de cuenca (Decreto por el que se reforman, adicionan y derogan diversas disposiciones de la Ley de Aguas Nacionales, 2004). ${ }^{9}$ Las atribuciones de la Conagua van desde el desarrollo, actualización y aplicación del Plan Nacional Hídrico, hasta la implementación de programas específicos a nivel regional y de cuenca, lo que incluye el desarrollo de la infraestructura ambiental e hídrica en coordinación con los gobiernos locales y estatales. En lo que se refiere a la calidad del agua, la Conagua propone y aplica los estándares operativos bajo las normas oficiales mexicanas, las

\footnotetext{
${ }^{8}$ Algunas de estas reglamentaciones proporcionan mecanismos para proteger los recursos naturales y espacios abiertos. Como ejemplos a nivel federal figuran el Nacional Environmental Policy Act (NEPA), el Habitat Conservation Plan (HCP), el National Historic Preservation Act (NHPA), y el Endangered Species Act (ESA). A nivel estatal se encuentran el California Environmental Quality Act (CEQA), el California Endangered Species Act, y Natural Community Conservation Planning Act (NCCP); a nivel condado están la County of San Diego Biological Mitigation Ordinance y la County of San Diego Resource Protection Ordinance (RPO). Finalmente a nivel local se encuentra la City of San Diego Environmentally Sensitive Lands, la Resource Protection Ordinance, y el Associate Guidelines.

${ }^{9}$ Desde la promulgación de la Ley de Aguas Nacionales de 1992, los marcos institucionales federales en la materia han promovido el fortalecimiento de la cuenca como un ente formal de gestión para la planificación y gestión de los recursos hídricos en el territorio mexicano. En esta tendencia, la figura de los consejos de cuenca se presenta como un actor central de la política hídrica, integrando la presencia local y regional a la toma de decisiones en temas que afectan a la cuenca. Estos consejos establecen para su funcionamiento, el apoyo de comisiones y comités de cuenca a niveles de subcuenca y microcuenca, respectivamente. En el caso de Baja California solo existe una comisión de cuenca -la Comisión del Río Colorado- la cual apoya el trabajo del Consejo de Cuenca de Baja California.
} 
cuales son expedidas por la dependencia del gobierno federal, la Semarnat, ${ }^{10}$ de la que es un órgano.

Los esquemas anteriores tienen manifestaciones específicas al tratarse de cuencas transfronterizas entre ambos países. Por un lado, la legislación hídrica federal en México carece de regulación de cauces transfronterizos, ${ }^{11}$ así como de funciones o actividades para los consejos de cuenca en aspectos relacionados con ellas (Castro y Sánchez, 2005). Todo lo relacionado con cuencas internacionales queda en el ámbito de la Secretaría de Relaciones Exteriores, a través de la cila y de los términos del Tratado de Aguas de 1944. Los gobiernos estatales y locales deben avenirse, por otro lado, al mandato establecido en el artículo 117 fracción I de la Constitución mexicana, que impide concurrir formalmente en negociaciones internacionales de cualquier índole (González, 2005). En el caso de Estados Unidos, como se ha mencionado, el modelo político-administrativo federalizado permite el involucramiento de agencias no federales en los procesos de planeación y toma de decisiones, pudiendo reflejar estas los intereses de los gobiernos locales y regionales en temas que les atañen directamente. Una circunstancia que es importante hacer notar y que refuerza la capacidad de autonomía de los gobiernos subnacionales en la frontera estadounidense, es la relación de servicio que mantienen con la contraparte correspondiente de la cILA, lo que les permite tomar parte activa en la toma de decisiones sobre proyectos que beneficien a sus comunidades (Mumme, 1984).

Las diferencias estructurales anteriores han definido marcadamente el papel gubernamental en el desarrollo y efectividad de las iniciativas y programas binacionales que se han desarrollado en la CRT. En tanto que las instancias gubernamentales mexicanas han visto restringida mayormente su capacidad operativa, los gobiernos estatales y locales en el caso californiano han tenido una participación más directa y activa, y su apoyo se ha visto reflejado en proyectos como el Programa de Manejo Sustentable del Estuario del Río Tijuana (Trnerr, 2010), el Tijuana River Valley Recovery Strategy (Tijuana River Valley Recovery Team, 2012) y el control de sedimentos en el Cañón de Los Laureles en la zona urbana de Tijuana (Heyn et al., 2008). Asimismo, las instancias no federales de la parte estadounidense de la CRT han tenido un papel central, llevando la iniciativa en la búsqueda de soluciones cooperativas con sus contrapartes bajacalifornianas. Ejemplos de esto último son совRо у el Consejo Fronterizo del Agua (вwс), ambos dependientes de sANDAG (Brown et al., 2003). En el caso de las iniciativas que han surgido de ámbitos no gubernamentales como fue el proyecto de creación del BWAC, la misma naturaleza federalizada del sistema político en California facilitó considerablemente la implementación de algunas de las recomendaciones originadas en dichos foros, entre ellas la formación del Grupo de Trabajo de Agua, como parte del Programa Frontera 2012, el subgrupo de trabajo de Ecología y el Comité Técnico del Agua creado bajo el Mecanismo de Enlace Fronterizo (Castro y Ganster, 2012).

El contexto anterior influye también en el actuar de la cila-IBwC a ambos lados de la frontera. Por un lado, la sección estadounidense se ha caracterizado por tener una

\footnotetext{
${ }^{10}$ Otra legislación federal en México relevante en temas ambientales es la Ley General del Equilibrio Ecológico y la Protección al Ambiente (LGEEPA).

${ }^{11}$ La única referencia a lo transfronterizo internacional en la actual Ley de Aguas Nacionales (2016, artículo 9, fracción IX) se refiere a la intervención de Conagua en asuntos del ámbito federal que tengan repercusión en acuerdos y tratados internacionales en cuencas transfronterizas.
} 
interacción activa con instancias y actores a los niveles locales y estatales para abordar la problemática ambiental de la cuenca. De esto dan cuenta, por ejemplo, los foros ciudadanos promovidos por el organismo, cuya representación en el área de San Diego viene funcionando desde 2002 con el objetivo de mantener un flujo constante de información entre la Comisión y el público en general, grupos ambientalistas, agencias gubernamentales y gobiernos locales entre otros (cila Sección Norteamericana, s.f). Entre las actividades realizadas por este foro están el seguimiento a proyectos como la Planta Internacional de Tratamiento de Aguas Residuales del Sur de la Bahía (South Bay International Wastewater Treatment Plant [sBIWTP]); el Proyecto de Control de Avenidas del Río Tijuana y el Grupo de Restauración del Valle del Río Tijuana. En el caso de México, las funciones de la cila se avienen estrictamente al mandato federal, lo que le resta flexibilidad operativa, y sus agendas y acciones en diferentes casos han estado desfasadas de las de su contraparte estadounidense. Es muy sintomático, en este sentido, el hecho de que tardaron más de 10 años en instituirse los foros ciudadanos por parte de la sección mexicana de la cILA, siguiendo una estructura y funciones parecidas a sus similares estadounidenses (CILA Sección Mexicana, 2014)..$^{12}$

Aunados a los problemas sustantivos que se han mencionado, existen otros desafíos operativos, tanto administrativos como de manejo, que obstaculizan la coordinación y comunicación gubernamental binacional en torno a la problemática hídrica y que requieren de una convergencia y acuerdos por parte de las autoridades responsables en ambos países. Tal es el caso del aumento en las medidas de seguridad fronteriza por parte del gobierno federal estadounidense a raíz de los eventos de septiembre 11, 2001, las que han implicado restricciones en términos de demoras y dificultades de programación a los esfuerzos binacionales de monitoreo y evaluación. Entre los casos más reiterados está la necesidad de documentación migratoria específica que facilite en un momento dado una interacción transfronteriza expedita.

\section{Algunas reflexiones}

Como se ha expuesto a lo largo de este trabajo, la CRT representa sin duda un caso único en el panorama de manejo binacional del agua entre México y Estados Unidos. A pesar de la ausencia de un mandato específico que distribuya binacionalmente las aguas del sistema del Río Tijuana, su cuenca ha sido objeto de múltiples iniciativas locales, producto mayormente de la preocupación y organización de los sectores no gubernamentales en torno a su problemática ambiental, particularmente la relacionada con el manejo del agua, las que constituyen una base de conocimiento y capacidades locales cuya utilidad ha sido reconocida y apoyada en forma diferenciada por los niveles subnacionales en cada país. En el caso de California, esta experiencia eventualmente atrajo el apoyo gubernamental a los niveles locales y regionales, con el financiamiento y seguimiento de diferentes proyectos. En contraste, la participación de las contrapartes mexicanas ha estado siempre condicionada por las características estructurales del sistema político administrativo vigente en el país y, por ende, sin la capacidad y autonomía para establecer compromisos específicos.

\footnotetext{
${ }^{12}$ Los primeros foros ciudadanos de la sección mexicana de la CILA se instalaron en 2014 en las ciudades de Tijuana y Nuevo Laredo.
} 
La experiencia de la CRT es relevante en el marco de las tendencias actuales en las formas de visualizar y abordar las problemáticas inherentes a los recursos hídricos compartidos transnacionalmente. El crecimiento urbano de la cuenca —básicamente en la parte mexicana - y la problemática ambiental que genera binacionalmente, son una muestra de las condiciones que existen hoy en día en regiones similares, cuya complejidad impone nuevos desafíos para modelos de política hídrica centralizados y limitados al territorio nacional. En la actualidad, esta visión se está modificando. Entre los factores detrás de dicho proceso se han identificado los cambios en los sistemas políticos y económicos internacionales, y sus efectos en las percepciones tradicionales sobre soberanía y cooperación (Blatter et al., 2001; Elhance, 2000), así como la importancia en sí que han cobrado los cursos internacionales de agua, como los espacios de estudio de las condiciones y problemáticas que inciden en las formas de gestión, y la identificación de escenarios de conflicto o cooperación (Giordano, Giordano y Wolf, 2002; Haftendorn, 2000; Uitto y Duda, 2002; Wolf, 1998). Un resultado de estos cambios ha sido una mayor participación de actores en los ámbitos inmediatos a las cuencas hídricas internacionales, permitiendo el establecimiento de nexos con las contrapartes a esos niveles.

En el caso de la frontera México-Estados Unidos, el contexto mencionado y sus capacidades potenciales han sido reconocidos implícitamente en investigaciones recientes sobre la viabilidad conceptual de modelos binacionales de cooperación a nivel de cuenca hidrológica, particularmente la región Tijuana-San Diego (Brown, 2002; Brown y Mumme, 1999; 2000). Asimismo, instancias gubernamentales como la Junta Ambiental del Buen Vecino (Good Neighbor Environmental Board [GNEB]), un órgano consultivo del Ejecutivo y Congreso estadounidenses, han planteado el manejo binacional del agua a partir del enfoque de cuenca bajo las consideraciones anteriores (GNEB, 2000).

Desde la perspectiva de las instituciones mexicanas, la experiencia descrita en la CRT motiva la reflexión sobre los beneficios potenciales que implica reconocer la importancia de los actores sociales y gubernamentales en los espacios subnacionales como intérpretes y facilitadores de los objetivos y alcances de la política hídrica federal en un marco de cooperación binacional, y de la necesidad de que exista una apertura operativa que los reconozca y fortalezca. En el caso de la CRT por ejemplo, los grupos y actores que han participado en las iniciativas binacionales descritas por la parte mexicana, han incluido a personas con alguna relación o conocimiento de las actividades y agendas de Conagua, a través de sus instancias encargadas de aplicar la política hídrica a estos niveles (Castro y Ganster, 2012), un recurso que está evidentemente subvaluado. Ciertamente, el Consejo de Cuenca de Baja California cuenta en sus sesiones con la presencia de un representante de la cila, pero se trata de una de las instancias invitadas sin voz ni voto, y su participación es esporádica e informativa (Castro y Sánchez, 2005).

La reciente firma del Acta 320 de la CILA-IBWC es sin duda un reconocimiento a la labor de los grupos y actores no gubernamentales en ambas partes de la CRT, en pro de la creación de un ente binacional para coordinar el manejo compartido de la cuenca (Saxod et al., 2007). Sin embargo, su futuro operativo impone desafíos. El primero tendrá que ver con la disposición y compromiso que muestren las partes gubernamentales mexicanas para el abordaje de problemas específicos en torno a los recursos hídricos de la CRT, tanto desde la perspectiva de flexibilizar la naturaleza rígida y burocrática de los operadores de la política hídrica en el país —nacional y 
transfronteriza-, como de su disposición a compartir información y recursos para la consecución de los proyectos específicos que identifique prioritariamente el Grupo Base Binacional (GBB). De la observación de estas dos condiciones dependerá en mucho el apoyo futuro de las contrapartes estadounidenses, en términos de continuar o aumentar los subsidios financieros con que han participado hasta ahora. Otro punto central tendrá que ver con el nivel de interacción y participación que tendrán los representantes no gubernamentales en el seno del GBB en la práctica, pues será fundamental que los grupos civiles puedan transmitir a esa instancia sus preocupaciones y propuestas en torno a las problemáticas de la CRT.

El Acta 320 plantea una vía interesante para que las instancias gubernamentales mexicanas incursionen en la búsqueda de mecanismos operativos que les faciliten una interacción abierta y dinámica con sus contrapartes sociales y gubernamentales al nivel subnacional. En tanto esto no exista, y quede únicamente en el ámbito de la cila la participación de México en estas iniciativas locales, se estará perdiendo no solo un recurso a nivel nacional, sino la oportunidad de avanzar en modelos de manejo cooperativo y sustentable de los recursos hídricos compartidos en la frontera norte de México.

\section{Referencias}

Blatter, J., Ingram, H. y Doughman, P. M. (2001). Emerging Approaches to Comprehend Changing Global Contexts. En J. Blatter y H. Ingram (Eds.), Reflections on Water. New Approaches to Trans-Boundary Conflicts and Cooperation (pp. 3-29). Cambridge, Reino Unido: мIт Press.

Brown, C. (2002). Research Into Binational Watershed Councils (Consejos Binacionales de las Cuencas) as Instruments for Conflict Resolution in the Upper Santa Cruz Watershed. Arizona, Estados Unidos de América: Udall Center for Studies in Public Policy.

Brown, C., Castro, J. L., Lowery, N. y Wright, R. (2003). Comparative Analisis of Transborder Water Management Strategies: Case Studies on the U.S.-Mexican Border. En S. Michel (Ed.), Binational Water Management Planning: Opportunities, Costs, Benefits, and Uninentended Consequences. Secure and Sustainable Water by 2020 (pp. 279-362). California, Estados Unidos de América: Southwest Center for Environmental Research and Policy.

Brown, C. y Mumme, S. P. (1999). Consejos de Cuencas: An Institutional Option for Transboundary Water Management on the U.S.-Mexico Border. International Review of Comparative Public Policy, 11, 115-140.

Brown, C. y Mumme, S. P. (2000). Applied and Theoretical Aspects of Binational Watershed Councils (Consejos de Cuencas) in the U.S.-Mexico Borderlands. Natural Resources Journal, 40(4), 895-929.

Castro, J. L. y Ganster, P. (7 de diciembre de 2012). Participación local transfronteriza en la frontera México-Estados Unidos: El Consejo Consultivo de la Cuenca Binacional del Río Tijuana. Tercer Congreso de la Red Temática del Agua de Conacyt, Ciudadanos y Sustentabilidad del Agua en México, México, Distrito Federal.

Castro, J. L. y Sánchez, V. (2005). La experiencia de un consejo de cuenca en un contexto binacional: el Consejo de Cuenca de Baja California. En S. Vargas y E. 
Mollard (Eds.), Problemas Socio-Ambientales y Experiencias Organizativas en las Cuencas en México (pp. 316-330). México: Instituto Mexicano de Tecnología del Agua.

City of San Diego. (2010). Plan de Manejo de Agua Urbana, 2010. Recuperado de https://www.sandiego.gov/sites/default/files/legacy/water/pdf/uwmp2010.pdf

Coastal. (2006). Tijuana River Estuary, State of the Critical Coastal Areas Report No. 80, junio 6. Recuperado de https://www.coastal.ca.gov/nps/Web/cca_pdf/socoastpdf/CCA80TijuanaRiverEstuary.pdf

Comienza última etapa de canalización del Arroyo Alamar. (30 de enero de 2015). Zeta, Libre como el Viento. Recuperado de http:/ /zetatijuana.com/2015/01/30/ comienza-ultima-etapa-de-canalizacion-del-arroyo-alamar/

Comisión Estatal del Agua de Baja California (CEA). (2008). Programa Estatal Hídrico del Estado de Baja California, 2008-2013. Recuperado de https://futurocostaensenada.files.wordpress.com/2010/02/peh20082013.pdf

Comisión Estatal de Servicios Públicos de Tijuana (CESPT). (2003). Plan Maestro de Agua Potable y Saneamiento en los Municipios de Tijuana y Playas de Rosarito. México: Autor.

Comisión Internacional de Límites y Aguas (ciLA). (1965). Acta 222, Conexión de Emergencia del Sistema de Alcantarillado de la Ciudad de Tijuana, Baja California, al Sistema Metropolitano de Alcantarillado de la Ciudad de San Diego, California. Ciudad Juárez, Chihuahua, 30 de noviembre de 1965. Recuperado de http://www.cila. gob.mx/actas/222.pdf

Comisión Internacional de Límites y Aguas (CILA). (1985). Acta 270, Recomendaciones para la primera etapa de las obras de alejamiento y tratamiento para la solución del problema fronterizo de saneamiento en Tijuana, Baja California/San Diego, California, Ciudad Juárez, Chihuahua, 30 de abril de 1985. Recuperado de http://www. cila.gob.mx/actas/270.pdf

Comisión Internacional de Límites y Aguas (CILA). (1990). Acta 283, Plan conceptual para la solución internacional del problema fronterizo de saneamiento en Tijuana, Baja California - San Diego, California. Aprobación: México - Agosto 8, 1990, Estados Unidos - Agosto 8, 1990, El Paso, Texas, 2 de julio de 1990. Recuperado de http:// www.cila.gob.mx/actas/283.pdf

Comisión Internacional de Límites y Aguas (CILA). (2015). Acta 320, Marco general para la cooperación binacional en los asuntos transfronterizos de la Cuenca del Rio Tijuana, Tijuana, Baja California, 5 de octubre de 2015. Recuperado de http://www.cila. gob.mx/actas/320.pdf

Comisión Internacional de Límites y Aguas Sección Mexicana (crla Sección Mexicana). (2014). Integración de la primer mesa directiva del foro ciudadano. Región de Tijuana, B.C. Recuperado de http://web.archive.org/web/20141203113100/ http://www.sre.gob.mx/CILAnorte/images/stories/pdf/mesadt.pdf

Comisión Internacional de Límites y Aguas Sección Norteamericana (cila Sección Norteamericana). (s.f). UsIBWC Citizens Forum in San Diego. Recuperado de http:/ / www.IBwC.gov/Citizens_Forums/CF_SBIWTP.html

Consejo Nacional de Población (Conapo). (2013). Proyecciones de la población de los municipios que componen las zonas metropolitanas, 2010-2030. Recuperado de http:// conapo.gob.mx/es/CONAPO/Proyecciones

Elhance, A. (2000). Hydropolitics: Grounds for Despair, Reasons for Hope. International Negotiation, 5(2), 201-222. 
Environmental Protection Agency (EPA). (2010). Border 2012 Information Database. Recuperado de http:/ /www.usmexicoborder2012.org

Friedkin, J. F. (1965). Proposed International Food Control Project, Tijuana River Basin in California and Baja California. Estados Unidos de América: International Boundary and Water Commission (IBWC).

Giordano, M., Giordano M. y Wolf, A. (2002). The Geography of Water Conflict and Cooperation: Internal Pressures and International Manifestations. The Geographical Journal, 168(4), 293-312.

González, M. (2005). La internacionalización de la frontera México-Estados Unidos en el marco legal. En A. A. Cortez, S. Whiteford y M. Chávez (Eds.), Seguridad, agua y desarrollo: El futuro de la frontera México-Estados Unidos (pp. 233-249). México: El Colegio de la Frontera Norte.

Good Neighbor Environmental Board (GNEB). (2000). Fourth Report of the Good Neighbor Environmental Board to the President and Congress of the United States. Recuperado de https://www.epa.gov/sites/production/files/documents/annrpt900eng.pdf

Haftendorn, H. (2000). Water and international conflict. Third World Quarterly, 21(1), 51-68.

Heyn, K., Keane-Dengel, R., Lewis, W., Phillips, J. y Virgilio, N. (2008). Mitigation of Impaired Stormwater Quality in Los Laureles Canyon, Tijuana, Mexico, Santa Barbara, California (Tesis de maestría). Recuperado de http://www.bren.ucsb.edu/ research/documents/TijuanaReport.pdf

Institute for Regional Studies of the Californias (IRSC). (2005). A Binational Vision for the Tijuana River Watershed. Recuperado de https://www.waterboards.ca. gov/sandiego/water_issues/tijuana_river_valley_strategy/docs/previous_meetings/2005_Tijuana_River_Watershed_Binational_Vision.pdf

McBride, R. (1981). The United States and Mexico: The Shape of the Relationship. En R. H. McBride (Ed.), Mexico and the United States (pp. 1-30). Nueva Jersey, Estados Unidos de América: Prentice Hall.

Michel, S. (2001). The Alamar River Corridor: An Urban River Park Oasis in Tijuana, Baja California, Mexico. California, Estados Unidos de América: Institute for Regional Studies of the Californias, San Diego State University.

Mumme, S. P. (1984). Regional Power in National Diplomacy: The Case of the U.S. Section of the International Boundary and Water Commission. Publius, 14(4), 115-135.

Mumme, S. P. y Collins, K. (2014). The La Paz Agreement 30 Years On. The Journal of Environment and Development, 23(3), 303-330.

Mumme, S. P., Collins K. y Castro J. L. (2014). Strengthening Binational Management of the Tijuana River. Water Law Review, 17(2), 329-357.

North American Development Bank (NADBank). (2013). 2013 Annual Report. Recuperado de http://www.nadb.org/pdfs/publications/2013AnnualReport.pdf

Saldaña, L. (2003). From Litigation to Legislation: Challenges to Binational Water Infrastructure Development in the San Diego-Tijuana Bioregion. Journal of Environment and Development, 12(4), 430-444.

Samaniego, M. A. (2006). Ríos internacionales entre México y Estados Unidos: los Tratados de 1906 y 1944. México: El Colegio de México. 
Saxod, E., Castro, J. L., Silvan, L. y Reyna, M. A. (2007). A Binational Planning Approach for the Development of the Tijuana River Watershed: Policy Options from Rhetoric to Action. Recuperado del sitio de Internet de Southwest Consortium for Environmental Research and Policy (scerp). http://trw.sdsu.edu/English/Projects/ Docs/ABinationalPlanningApproachfortheDevelopmentoftheTijuanaRiverWatershedReportFINAL.pdf

Spalding, M. (1999). Governance Issues under the Environmental Side Agreement of the NAFTa. En M. J. Spalding (Ed.), Sustainable Development in San Diego-Tijuana. Environmental, Social, and Economic Implications and Interdependence (pp. 57-71). California, Estados Unidos de América: Universidad de California San Diego.

Tijuana River National Estuarine Research Reserve (TRNERR). (2010). Comprehensive Management Plan for Tijuana River NERR and Tijuana Slough NWR, and Border Field State Park. Recuperado de http://trnerr.org/about/management-plan/ _

Tijuana River Valley Recovery Team. (2012). Recovery Strategy: Living with the Water. Recuperado de https://www.waterboards.ca.gov/sandiego/water_issues/tijuana_river_valley_strategy/docs/Recovery_Strategy_Living_with_the_Water.PDF

Trejo, C. y Castro, J. L. (2013). Gestión ambiental local. Los esfuerzos por rehabilitar el arroyo Alamar en Tijuana, Baja California. En V. Sánchez (Ed.), El arroyo Alamar de Tijuana. Un río urbano amenazado (pp. 113-146). México: Red Nacional de Investigación Urbana.

Uitto, J. y Duda, A. (2002). Management of Transboundary Water Resources: Lessons from International Cooperation for Conflict Prevention. The Geographical Journal, 168(4), 365-378.

Wolf, A. (1998). Conflict and cooperation along international waterways. Water Policy, $1(2), 251-265$.

\section{Material legislativo}

Decreto por el que se reforman, adicionan y derogan diversas disposiciones de la Ley de Aguas Nacionales. 29 de abril de 2004. Diario Oficial de la Federación. Recuperado de http://www.diputados.gob.mx/LeyesBiblio/ref/lan.htm

Ley de Aguas Nacionales. Artículo 9, fracción Ix. 24 de marzo de 2016. Diario Oficial de la Federación.

Tratado sobre distribución de aguas internacionales celebrado entre los Estados Unidos Mexicanos y los Estados Unidos de América. 3 de febrero de 1944. Recuperado de http://www.semarnat.gob.mx/archivosanteriores/temas/internacional/norte/Documents/Marco\%20Legal/003_Mex-EUA_Tratado_Distribución_Aguas_Internacionales\%201944.pdf

José Luis Castro Ruiz

Mexicano. Doctorado en Planeación Urbana y Regional, Universidad del Sur de California, E.U.A. Profesor-investigador de El Colegio de La Frontera Norte, Departamento de Estudios Urbanos y del Medio Ambiente en Monterrey, Nuevo León. Líneas de investigación: desarrollo urbano fronterizo, gestión urbana y transfronteriza del agua. Publicación reciente: Sustentabilidad ambiental y planeación estatal en la frontera 
norte de México: el caso de los recursos hídricos, en coautoría con María Eugenia González Ávila, El Colef y Cibnor (en prensa).

Stephen P. Mumme

Estadounidense. Doctorado en Ciencia Política, Universidad de Arizona, Estados Unidos. Profesor de Ciencia Política, Departamento de Ciencia Política de la Universidad Estatal de Colorado. Líneas de investigación: política ambiental comparativa, manejo ambiental en la frontera México-Estados Unidos, manejo ambiental transfronterizo en Norteamérica. Publicación reciente: (2017). Constructing Equity during the Salinity Crisis (1961-1973): Mexico, the United States and the Politics of Sharing on the Colorado River. Journal of the Southwest.

Kimberly Collins

Estadounidense. Doctorado en Ciencias Sociales Aplicadas, El Colegio de la Frontera Norte, México. Profesor Asociado de Administración Pública, Universidad Estatal de California, San Bernardino. Líneas de investigación: gobernanza local y planeación, globalización y redes sociales; Liderazgo, democracia y participación comunitaria. Publicación reciente: (2017). Globalization, Democracy, and Public Space: The Case of the U.S.-Mexican Border Region. Journal of Public Management and Social Policy. 